
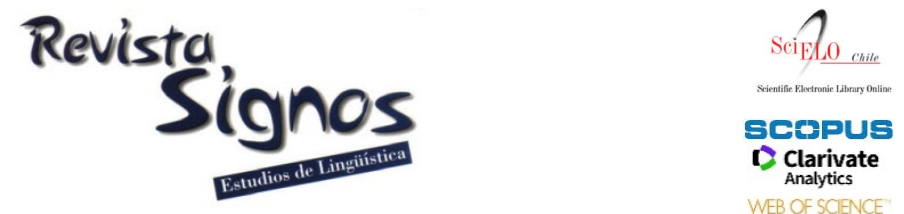

\title{
Diferencias en el dominio de la organización discursiva y en el uso de recursos léxico- gramaticales en explicaciones científicas producidas por estudiantes de $4^{\circ}$ básico
}

\section{Differences in the mastery of organizational features and in the use of lexical-grammatical resources in scientific explanations by fourth graders}

\author{
Alejandra Rubio N. \\ Pontificia Universidad CATÓLICA De CHILE \\ CHILE \\ avrubio@uc.cl
}

\author{
Alejandra Meneses A. \\ Pontificia Universidad Católica de CHILE \\ CHILE \\ amenesea@uc.cl
}

Received: 13-V-2019 / Accepted: 13-VII-2020

DOI: $10.4067 /$ S0718-09342021000200438

\section{Resumen}

Si bien existen estudios sobre recursos discursivos y léxico-gramaticales utilizados en explicaciones producidas por escolares, menos investigación se ha desarrollado en español sobre estos en géneros disciplinares escritos por estudiantes de educación primaria. En el presente artículo, se describen, comparan y relacionan la organización discursiva, los recursos asociados a la escritura -longitud medida en cantidad de palabras y cláusulas, diversidad léxica y complejidad sintáctica- y aquellos recursos específicos del lenguaje académico -vocabulario académico, vocabulario científico, nominalizaciones y nexos causales- presentes en explicaciones científicas producidas por estudiantes chilenos de $4^{\circ}$ básico de tres colegios pertenecientes a distintos grupos socioeconómicos. Se transcribieron y analizaron 326 explicaciones usando CLAN (Computerized Language Analysis) para las medidas automáticas y semiautomáticas. Las explicaciones se compararon de acuerdo a las siguientes variables: sexo, grupo socioeconómico y tema de la explicación. Para el análisis de datos se aplicaron pruebas $t$ y ANOVA multifactorial. Los hallazgos de esta investigación dan cuenta de diferencias significativas en el uso de distintos recursos lexicogramaticales al ser comparados según las variables mencionadas. Respecto a la organización discursiva, las explicaciones producidas por los estudiantes integran afirmaciones, pero no evidencias, siendo esta última la fase discursiva que distingue este género disciplinar. En consecuencia, la presente investigación releva la necesidad de diseñar propuestas de enseñanza que 
promuevan el desarrollo de habilidades de escritura epistémica en el contexto escolar para así fomentar el aprendizaje del lenguaje académico y de la literacidad disciplinar.

Palabras Clave: Explicaciones científicas, escritura epistémica, lenguaje académico, organización discursiva, literacidad disciplinar.

\begin{abstract}
Although there are studies about discursive and lexicogrammatical resources displayed in explanations produced by school students, less research has been developed about them in Spanish on disciplinary genres written by primary school students. In this article we will describe, compare and relate discursive organization, resources linked to writing -length measured in amount of words and clauses, lexical diversity and syntax complexity- and those resources specific to academic language -academic vocabulary, scientific vocabulary, nominalizations and causal links- included in scientific explanations produced by Chilean fourth-graders of three schools from different socioeconomical groups. Three hundred twenty-six explanations were transcribed and analyzed using CLAN (Computerized Language Analysis) for the automated and semiautomated measures. The explanations were compared according to the following variables: sex, socio-economical group, and topic of the explanation. Tand ANOVA multifactorial tests were applied for the data analysis. The findings show significant differences in the use of different lexicogrammatical resources when compared using the mentioned variables. Regarding discursive organization, the explanations produced by the students integrate affirmations, but not evidence, the latter being the distinctive discursive phase of this disciplinary genre. Consequently, this research highlights the need to design explicit teaching proposals that promote the development of epistemic writing skills in the school context in order to foster the learning of academic language and disciplinary literacy.
\end{abstract}

Key Words: Scientific explanation, epistemic writing, academic language, discursive organization, disciplinary literacy.

\title{
INTRODUCCIÓN
}

La escritura como habilidad comunicativa y práctica discursiva juega un papel fundamental en el aprendizaje escolar, pues permite no solo la comunicación en contextos diversos sino también el desarrollo de habilidades de pensamiento crítico y la construcción de conocimientos en distintas disciplinas (Miras, 2000; Ravid \& Tolchinsky, 2002; Graham, Gillespie \& McKeown, 2013). Si bien en los últimos años ha crecido la investigación en escritura, la investigación empírica sigue siendo menor en comparación con los estudios sobre comprensión lectora (Miller \& McCardle, 2011). La revisión de la investigación nacional sobre escritura realizada en las dos últimas décadas por Bañales, Ahumada, Martínez, Martínez y Messina (2018) concluyó que esta se ha enfocado, principalmente, en la narración, mientras que los textos expositivos y de opinión han sido menos investigados.

La evaluación nacional estandarizada sobre escritura para escolares de $6^{\circ}$ básico ha demostrado que las mujeres logran producir textos escritos de mayor calidad en comparación con los hombres y que los estudiantes que producen textos de mejor 
calidad pertenecen a los niveles socioeconómico alto (Agencia de Calidad de la Educación, 2017). Estos resultados son coherentes con los hallazgos de Sotomayor, Gómez, Jéldrez, Bedwell, Domínguez y Ávila (2016), quienes evaluaron la calidad de tres géneros discursivos producidos por 687 estudiantes chilenos de $4^{\circ}$ básico y encontraron que los desempeños más altos fueron obtenidos en cuentos en comparación con las cartas y las noticias. Además, concluyeron que existen diferencias significativas según sexo y grupo socioeconómico: las mujeres obtienen un desempeño significativamente más alto que los hombres en todos los géneros producidos, así como los estudiantes de grupo socioeconómico alto tienen un desempeño mayor en escritura que los estudiantes de otros grupos. Sin embargo, menos estudios se han focalizado en caracterizar los recursos que operan al nivel discursivo y lingüístico en la producción de diferentes géneros, objetivo relevante para comprender el papel que estos recursos desempeñan en el desarrollo y aprendizaje de las habilidades de escritura. Aravena, Figueroa, Quiroga y Hugo (2016) describieron la organización discursiva de historias personales y artículos explicativos producidos por estudiantes de entre 12 a 16 años y encontraron que a medida que los estudiantes avanzan en edad producen narraciones en que aumenta la evaluación; sin embargo, no encontraron diferencias en la organización de las explicaciones. Por su parte, Aravena y Hugo (2016) exploraron los recursos sintácticos en estos dos géneros y encontraron diferencias significativas según género discursivo, sexo y nivel socioeconómico.

La escritura no solo es relevante para aprender a construir significados en distintos contextos sino que es también una práctica habitual de aprendizaje y evaluación en todas las disciplinas escolares. No obstante, son escasas las investigaciones empíricas sobre los recursos discursivos y léxicogramaticales usados por escolares para la producción de un género disciplinar (Ibáñez, 2010) y aún menos estudios se han realizado sobre estos en contextos escolares de educación primaria (Ávalos, Gómez, Gort \& Secada, 2017; Meneses, Hugo, Montenegro, Valenzuela \& Ruiz, 2018). El objetivo de este estudio descriptivo-correlacional es describir, comparar y relacionar los modos de organización discursiva, los recursos léxico-gramaticales asociados a la escritura y los recursos específicos de lenguaje académico usados por estudiantes de $4^{\circ}$ básico en explicaciones científicas.

Desde una perspectiva social, enfocada en la escritura y la literacidad disciplinar (Shanahan \& Shanahan, 2008; Bazerman, Little, Bethel, Chavkin, Fouquette, \& Garufis, 2016) y desde una aproximación funcional del lenguaje (Berman \& Ravid, 2009; Snow \& Uccelli, 2009), en particular, desde una teoría sociocultural sobre el desarrollo del lenguaje para el aprendizaje (Snow \& Uccelli, 2009; Uccelli, 2019), esta investigación tiene como propósito hacer una contribución empírica al campo de la escritura epistémica y explorar las diferencias en los recursos discursivos y lingüísticos usados por estudiantes de $4^{\circ}$ básico según su sexo y grupo socioeconómico y según las temáticas desarrolladas, así como determinar las relaciones entre estos distintos 
recursos que los estudiantes ponen en funcionamiento cuando escriben. Esta investigación estudia las variaciones en los repertorios discursivos y lingüísticos de estudiantes de $4^{\circ}$ básico con el fin de comprender cómo estos recursos operan en un género disciplinar producido por escolares que ya no solo aprenden a producir textos sino también escriben para aprender. De este modo, se espera contribuir con evidencia para informar a intervenciones que puedan andamiar el crecimiento del lenguaje en edades escolares y a estudios que busquen comprender cómo estas habilidades y conocimientos discursivos y lingüísticos se relacionan entre sí para en estudios posteriores investigar sobre su contribución en el desarrollo de las habilidades de escritura en interacción con conocimientos disciplinares.

\section{Marco teórico}

\subsection{Explicaciones en ciencias}

Existen distintas aproximaciones teóricas para abordar el estudio de las explicaciones. Desde una perspectiva discursiva, Bronckart (2004) la caracteriza como una secuencia que se organiza a partir de la problematización de un fenómeno no cuestionable que se presenta de manera no completa y necesitado de desarrollo para lo que se presentan causas o razones que lo reformulan. Desde la Lingüística Sistémico Funcional, Brisk (2015) caracteriza las explicaciones como un género escolar que los estudiantes de educación primaria aprenden a lo largo de la escolaridad para el aprendizaje en las áreas de ciencias e historia y las categoriza según el modo de organización de la información en 'factorial', 'secuencial', 'cíclica', 'causal', entre otras. Por su parte, desde la enseñanza de las ciencias, Andrade, Freire y Baptista (2017) plantean que aún no existe consenso sobre cómo definir la explicación científica y diferenciarla de la argumentación. Este estudio adopta una perspectiva sociocultural y se interesa, en particular, en el territorio de la literacidad disciplinar (Bazerman et al., 2016; Shanahan \& Shanahan, 2008); por lo tanto, se operacionaliza la explicación científica considerando las evidencias levantadas a partir de investigación en el área de enseñanza de la ciencias (McNeill, Lizotte, Krajcik \& Marx, 2006; Krajcik \& Sutherland, 2010; McNeill \& Krajcik, 2012; Andrade et al., 2017; Ávalos et al., 2017). En consecuencia, para este estudio se define la explicación científica como un género disciplinar que tiene como finalidad explicitar las relaciones causales de un fenómeno científico sustentadas en evidencias que apoyen dicha relación.

En los actuales estándares estadounidenses de enseñanza de las ciencias, se ha establecido la explicación científica como una práctica auténtica que todo estudiante debe aprender a lo largo de su escolaridad (National Research Council, 2012), pues aumenta la comprensión de los fenómenos naturales, ya que los estudiantes deben otorgar sentido a lo que sucede a su alrededor (National Research Council, 2012) y, además, los aproxima a la naturaleza del conocimiento científico (Andrade et al., 
2017). Más aún, dicho marco establece que los estudiantes tempranamente deberían aprender a construir explicaciones científicas partiendo por la observación y establecimiento de relaciones causales de fenómenos directamente observables (K-2) pasando por la construcción de modelos simples basados en evidencia que expliquen fenómenos observables (3-5) y llegando a modelos abstractos y detallados de fenómenos más complejos y no directamente observables (6-12) National Research Council, 2012). Dos elementos distinguen a la explicación científica de otros géneros: la construcción de relaciones causales y la incorporación de datos que funcionan como evidencias que sustenten dichas relaciones (McNeill et al, 2006; Krajcik \& Sutherland, 2010; McNeill \& Krajcik, 2012; Zembal-Saul, McNeill \& Hershberger, 2013). El marco propuesto por el National Research Council (2012) releva que las explicaciones científicas basadas en evidencias son escasamente enseñadas en la escuela y enfatiza su relevancia en la construcción de un conocimiento científico crítico.

Ahora bien, la producción de géneros disciplinares es una tarea altamente desafiante para los estudiantes porque no solo requieren de un conocimiento sobre un proceso científico abstracto y muchas veces no directamente observable, sino que también precisan de un manejo sobre la organización discursiva de la explicación científica, así como del uso de un lenguaje académico y científico que les permita desplegar las relaciones de causalidad sobre un determinado proceso (Seah, Clark \& Hart, 2011; Seah, 2015; Ávalos et al., 2017). En este sentido, la descripción y comparación de los recursos discursivos y lingüísticos en un género disciplinar producido por estudiantes parte del supuesto de que el papel del lenguaje no es transparente y, por lo tanto, este media la construcción del conocimiento sobre un fenómeno y las prácticas discursivas disciplinares (Schleppegrell, 2004; Grøver, Uccelli, Rowe \& Lieven, 2019). De este modo, resulta de interés caracterizar estos recursos y establecer relaciones entre ellos para comprender cómo operan en la construcción de explicaciones científicas, ya que el lenguaje académico y científico juega un rol relevante en el aprendizaje de principios, conocimientos y valores de las ciencias; es decir, aprender ciencias también significa aprender su lenguaje (Fang, 2004, 2014; Seah et al., 2011; Seah, 2015; Ávalos et al., 2017).

\subsection{Organización discursiva de las explicaciones científicas}

Aprender a producir explicaciones científicas conlleva conocimiento acerca de las fases discursivas o estrategias retóricas para desplegar las relaciones causales según demandas epistémicas propias de esta área de conocimiento (McNeill et al., 2006; Krajcik \& Sutherland, 2010; McNeill \& Krajcik, 2012). En el estudio de Ávalos et al. (2017) sobre explicaciones científicas producidas por estudiantes de $3^{\circ}$ grado, se analizaron las fases de introducción, afirmación y conclusión diferenciando las producciones por los desempeños obtenidos. Los resultados mostraron que en las producciones de bajo desempeño, los estudiantes construyeron un inicio ficcional más parecido a los utilizados en géneros narrativos literarios, mientras que en las 
explicaciones más logradas, los escritores incorporaron preguntas retóricas para involucrar a los lectores. Otra diferencia significativa entre ambos grupos fue la incorporación de una conclusión en las explicaciones de mayor logro en comparación con las de menor logro. Sin embargo, este estudio no identificó la evidencia como una fase discursiva fundamental de la explicación científica.

Desde la investigación sobre enseñanza de la ciencia, McNeill y Krajcik (2012) así como Zembal-Saul et al. (2013) han planteado que la explicación científica es una práctica auténtica que está conformada por las fases de 'afirmación', 'evidencia' y 'razonamiento'. Para este estudio se adopta la propuesta desarrollada desde la enseñanza de la ciencias (McNeill et al., 2006; Krajcik \& Sutherland, 2010; McNeill \& Krajcik, 2012). Como señalan McNeill y Krajcik (2012), la ‘afirmación' es una respuesta provisoria a la pregunta científica en la que se plantea una relación entre ideas que explicitan la causa de un fenómeno o proceso científico; la 'evidencia' se operacionaliza como los datos científicos seleccionados y que apoyan satisfactoriamente la afirmación y, por último, el 'razonamiento' es entendido como la fase discursiva en la que se explicitan los principios o relaciones de causa y efecto que explican un proceso. McNeill et al. (2006) demostraron que estudiantes estadounidenses de $7^{\circ}$ grado que recibieron distintos andamiajes para producir una explicación científica configurada por estas tres fases lograron avances significativos en la producción de sus explicaciones; sin embargo, el razonamiento fue la fase que presentó mayor dificultad para los estudiantes. McNeill y Krajcik (2012) realizaron adaptaciones para estudiantes de educación primaria e incorporaron andamiajes solo incluyendo la 'afirmación' y la 'evidencia'. En este sentido, la fase discursiva que caracteriza a la explicación científica es aquella referida a la incorporación de la evidencia, pues aporta información producida a través de alguna práctica científica -experimentación o construcción de modelos. Esta información se considera como datos científicos que respaldan la relación de causa-efecto propuesta en la afirmación.

\subsection{Recursos léxico-gramaticales asociados a la escritura}

Diversos estudios han abordado el problema de determinar qué recursos léxicogramaticales son predictores de calidad de la escritura en distintos géneros discursivos. Dichos estudios, además de determinar qué recursos léxico-gramaticales medir, determinan -de manera independiente- la calidad de la escritura mediante rúbricas con el fin de establecer las contribuciones específicas de los recursos en la calidad de los textos (Beers \& Nagy, 2009; McNamara, Crossley \& McCarthy, 2010; Uccelli, Dobbs \& Scott, 2013; Salas, Llaraudó, Castillo, Taulé \& Martí, 2016). En un estudio sobre textos persuasivos producidos por estudiantes de último año de secundaria, Uccelli et al. (2013) midieron la longitud de los textos en número total de cláusulas, diversidad léxica, diversidad de marcadores discursivos empleados y complejidad sintáctica. Los resultados mostraron que los marcadores organizacionales y 
epistémicos predijeron significativamente la calidad de las producciones de los estudiantes más allá de la contribución de otros recursos. Por otra parte, en un estudio sobre textos narrativos producidos por estudiantes de diversos niveles educativos (9, 12, 16 años y adultos), Salas et al. (2016) analizaron la diversidad léxica, la complejidad sintáctica y el uso de recursos cohesivos. Los modelos de predicción empleados entregaron resultados diferentes dependiendo de la edad de los participantes. En el caso de los estudiantes escolares, la complejidad sintáctica predijo la calidad (palabras por sintagma sustantivo para la muestra de estudiantes de 9 años y porcentaje de conjunciones subordinantes para la muestra de estudiantes de 16 años). En cambio, la diversidad léxica fue el predictor más significativo en el caso de los adultos.

Ya Beers y Nagy (2009) habían también realizado comparaciones entre distintas mediciones de complejidad sintáctica para predecir la calidad de géneros con secuencia explicativa y narrativa producidos por estudiantes de $8^{\circ}$ grado. Los resultados mostraron que el número de cláusulas por unidad $\mathrm{T}$ predice la calidad para las narraciones, mientras que en el caso de las explicaciones el número de palabras por cláusula contribuye a su calidad. Por su parte, McNamara et al. (2010) estudiaron las diferencias en cohesión y sofisticación lingüística en ensayos argumentativos producidos por estudiantes universitarios usando mediciones automáticas. Los resultados mostraron que los índices más predictivos de calidad en este género son la complejidad sintáctica, así como la diversidad y frecuencia léxica.

Los estudios mencionados dan cuenta del impacto de los recursos léxicogramaticales en la calidad de la escritura. Para el presente estudio, solo se determinará la frecuencia de uso de algunos de estos recursos léxico-gramaticales asociados en estudios previos a escritura: longitud medida como total de palabras y cláusulas, diversidad léxica y complejidad sintáctica. Esta decisión responde a la necesidad de realizar una descripción lingüística considerando una diversidad de recursos desplegados por estudiantes de $4^{\circ}$ básico en explicaciones científicas y profundizar en las variaciones de los recursos según sexo, nivel socioeconómico y temática. En futuros estudios se explorará sobre la contribución de estos recursos específicos en la calidad de la explicación científica.

Estos recursos se operacionalizan como 'recursos asociados a la escritura' para determinar, posteriormente, si existen diferencias significativas en el dominio de estos cuando se produce un género disciplinar, ya que la mayoría de los estudios revisados se han enfocado en la escritura de géneros de conocimiento general y no disciplinares (Salas et al., 2016).

\subsection{Recursos de lenguaje académico en explicaciones científicas}

Si bien el dominio de las fases discursivas y el manejo de los recursos léxicogramaticales asociados a la escritura son relevantes para la construcción de explicaciones científicas, los estudiantes también requieren aprender recursos léxico- 
gramaticales específicos del lenguaje académico y científico (Fang, 2004, 2014; Seah, 2015, Seah et al., 2011). Siguiendo a Schleppegrell (2004, 2012), el lenguaje académico se define como un registro usado en actividades de enseñanza y aprendizaje formal, es decir, corresponde a aquellos recursos que se utilizan en los procesos de escolarización. Uccelli, Phillips Galloway, Barr, Meneses y Dobbs (2015) definen operativamente el lenguaje académico como una constelación de recursos discursivos y lingüísticos de uso frecuente en los géneros académicos escolares en comparación con las interacciones cotidianas. A partir de una amplia revisión de estudios lingüísticos y pedagógicos, se sistematizaron los recursos más relevantes de los géneros académicos escolares para luego definir el conjunto de habilidades clave del lenguaje académico transdisciplinar (Uccelli et al., 2015), a saber: uso de nominalizaciones, conectores lógicos, anáforas conceptuales, marcadores epistémicos, entre otros.

Por consiguiente, los procesos de enculturación y escolarización de los estudiantes no solo implican el aprendizaje de nuevos conocimientos, sino también el aprendizaje de habilidades y recursos propios del registro académico escolar (Schleppegrell, 2004, 2012; Snow \& Uccelli, 2009; Bazerman et al., 2016; Uccelli, 2019). En este contexto, el lenguaje académico como registro se distancia del lenguaje cotidiano, pues requiere del dominio de recursos lingüísticos y discursivos que son usados con frecuencia en los contextos para el aprendizaje y la evaluación (Schleppegrell, 2004, 2012; Snow \& Uccelli, 2009; Uccelli et al., 2015; Uccelli, 2019). Es fundamental diferenciar el dominio del lenguaje académico de la verborrea que oscurece las relaciones entre las ideas; por el contrario, como plantea Uccelli (2019), el lenguaje académico es entendido como un registro que andamia los aprendizajes en contextos escolares, sobre el cual los estudiantes han tenido menos acceso desde su experiencia cotidiana y que requieren para avanzar en sus procesos de escolarización.

Desde una perspectiva funcional, la escritura científica permite construir y comunicar conocimiento, principios, creencias y formas de razonamiento disciplinar a partir de ciertas elecciones léxico-gramaticales apropiadas para estos contextos (Fang, 2004, 2014). El uso de los recursos propios del lenguaje científico permite teorizar y construir significados sobre fenómenos y procesos científicos, dada la relación existente entre la construcción del conocimiento y las elecciones lingüísticas para comunicar dicho saber (Fang, 2004, 2014; Seah et al., 2011; Seah, 2015,).

De acuerdo con lo planteado por Fang (2004, 2014), el lenguaje científico se caracteriza por una 'densidad informativa' que da cuenta del empaquetamiento y condensación de ideas, medida a través del número de palabras con contenido semántico por cláusulas o como el porcentaje del total de palabras con contenido semántico en un texto. Por otra parte, el lenguaje científico se caracteriza por un alto nivel de abstracción y una alta presencia de 'nominalizaciones' y ‘tecnicismos'. La 
nominalización es un recurso léxico-gramatical de conversión de procesos expresados por verbos en participantes expresados por sustantivos (Fang, 2004, 2014). El empleo de nominalizaciones permite crear palabras técnicas para establecer relaciones de causalidad, sintetizar información y nombrar fenómenos (Schleppegrell, 2012; Ávalos et al., 2017), mientras que los tecnicismos, también conocidos como vocabulario disciplinar, corresponden a la terminología de una disciplina especializada (Schleppegrell, 2004; Ávalos et al., 2017). Además, la escritura científica se caracteriza por el uso de conectores que permiten establecer relaciones lógicas entre las ideas construidas en una diversidad de géneros disciplinares (Schleppegrell, 2004; Seah, 2015; Ávalos et al., 2017). En este estudio se explorarán algunos de estos recursos léxico-gramaticales propios del lenguaje académico a saber: vocabulario académico, vocabulario científico, nominalizaciones y nexos causales.

\section{Propósito de este estudio}

La presente investigación es de tipo descriptivo-correlacional. Su objetivo es describir, comparar y relacionar la organización discursiva de la explicación científica, el uso de los recursos léxico-gramaticales asociados a la escritura -'longitud' medida a través del total de palabras y de cláusulas, 'diversidad léxica’ y 'complejidad sintáctica'y el uso de aquellos propios del lenguaje académico -vocabulario académico, vocabulario científico, nominalizaciones y nexos causales- en explicaciones científicas producidas sin intervención pedagógica por estudiantes chilenos de $4^{\circ}$ básico de tres colegios pertenecientes a distintos grupos socioeconómicos con el fin de realizar una descripción lingüística con orientación pedagógica.

Las preguntas que guían la presente investigación son dos:

(1) ¿Qué fases de la organización discursiva, recursos léxico-gramaticales asociados a la escritura y recursos específicos del lenguaje académico son utilizados por estudiantes de $4^{\circ}$ básico en explicaciones científicas escritas? ¿Existen diferencias según sexo y grupo socioeconómico de la institución educativa a la que pertenecen, así como sobre el tema que escriben?

(2) ¿Qué relación existe entre los patrones de uso de las fases de organización discursiva, los recursos léxico-gramaticales asociados a la escritura y los recursos del lenguaje académico en las explicaciones producidas por los estudiantes de $4^{\circ}$ básico?

\section{Marco metodológico}

El presente estudio se enmarca dentro de una investigación más amplia, cuyo objetivo general era mejorar el aprendizaje en el área de ciencias en estudiantes chilenos de $4^{\circ}$ básico a través de una intervención enfocada en la enseñanza explícita del lenguaje académico. Particularmente, este artículo busca caracterizar las 
explicaciones científicas producidas por estudiantes sin intervención de distintos colegios para realizar una descripción lingüística inicial que permita comprender las fases de organización discursiva, los recursos léxico-gramaticales asociados a la escritura y los recursos específicos del lenguaje académico usados por estudiantes de $4^{\circ}$ básico.

\subsection{Participantes}

En este estudio, participaron 163 estudiantes chilenos de $4^{\circ}$ básico de tres colegios ubicados en Santiago (Región Metropolitana) pertenecientes a distintos GSE según la Agencia de Calidad de la Educación (2013). El primer establecimiento, de dependencia municipalizada, pertenece a un GSE medio-bajo: la mayoría de los apoderados del establecimiento declaran tener entre 10 y 11 años de escolaridad y un ingreso que varía entre los 290.000 y los 460.00 mil pesos. Entre el 57,01\% y el 75\% de los estudiantes del establecimiento se encuentra en una condición de vulnerabilidad social.

El segundo colegio corresponde a un establecimiento particular subvencionado perteneciente a un GSE medio: la mayoría de los apoderados ha declarado tener entre 12 y 13 años de escolaridad y un ingreso entre los 460.001 y los 770.000 mil pesos. Entre el 35,01\% y el $57 \%$ de los estudiantes se encuentra en condición de vulnerabilidad.

El último colegio de la muestra, de dependencia particular, pertenece a un GSE alto: los apoderados han declarado tener 16 o más años de escolaridad y un ingreso del hogar de 1.460.001 o más. Asimismo, el 9\% o menos de los estudiantes se encuentra en condición de vulnerabilidad social.

En cuanto al sexo de los estudiantes, el 56\% corresponde al sexo masculino (92 hombres), mientras que el 44\%, al sexo femenino (71 mujeres).

\subsection{Tareas}

Para la construcción del corpus, se diseñaron dos tareas de producción de explicaciones científicas a partir de objetivos de aprendizaje establecidos en el currículum nacional. Dichas tareas -junto a otras que quedan fuera de esta investigación- fueron tomadas grupalmente por evaluadores capacitados en la modalidad de prueba escrita, con una duración total de 60 minutos y al término del año escolar, con el objeto de asegurar que todos contasen con la oportunidad de haber aprendido sobre los temas científicos. No obstante, estos estudiantes no recibieron enseñanza explícita sobre cómo producir explicaciones científicas pues solo se buscaba recolectar información inicial sobre recursos discursivos y lingüísticos usados por estudiantes chilenos en este género disciplinar. Se obtuvo el consentimiento de padres y estudiantes, así como la carta de autorización de los directivos de los establecimientos siguiendo los protocolos éticos pertinentes. 
Las preguntas para cada explicación científica fueron (1) ¿Cómo se junta el brazo con el antebrazo? y (2) ¿Cómo cambia una materia de estado sólido a líquido? Cada estudiante produjo dos explicaciones; por lo tanto, el corpus total quedó constituido por 326 producciones.

\subsection{Codificación}

Cada una de las explicaciones fue digitada y los errores ortográficos fueron corregidos con el fin de evitar el sesgo durante la codificación. Todas las producciones fueron transcritas usando el sistema CHAT (MacWhitnney, 2000) para luego realizar un análisis de medidas automáticas y semiautomáticas.

\subsection{Organización discursiva}

Cada explicación científica se segmentó a partir de la adaptación de las fases discursivas planteadas por McNeill y Krajcik (2012) y Zembal-Saul et al. (2013). Siguiendo los criterios de esta investigación, se desarrolló un manual de segmentación y codificación (Ruiz, Meneses, Hugo \& Rubio, 2018). En cada explicación segmentada se identificó la presencia de fases discursivas, pero no se determinó la calidad de la explicación ni la adecuación del contenido científico. Las fases discursivas consideradas para este estudio son:

(1) Afirmación: Es entendida como una respuesta provisoria a la pregunta científica planteada. En ella pueden ser mencionado el fenómeno científico por explicar ("una materia cambia de estado sólido a líquido" proceso + participantes) y la idea propuesta en la que desarrolla una causa a la que se le atribuye el surgimiento del fenómeno ("a causa del calor absorbido por un cuerpo a menor temperatura").

(2) Evidencia: Es operacionalizada como los datos científicos que apoyan la afirmación ("Basado en el experimento cuando un objeto a mayor temperatura está cerca del hielo este absorbe el calor del objeto y se derrite”).

El 20\% de las explicaciones de la muestra se sometió a una doble segmentación realizada por dos codificadores previamente entrenados. Posteriormente, se calculó el índice Kappa de Cohen para así asegurar la fiabilidad entre codificadores. Se obtuvo un resultado de 0,97 , lo que da cuenta de la confiabilidad de la segmentación. Adicionalmente, dos expertas realizaron una segunda revisión para asegurar la adecuada segmentación del 100\% de las explicaciones.

Una vez realizada la segmentación, se determinaron distintos niveles según los patrones de uso de las distintas fases en las explicaciones tomando en consideración la propuesta de McNeill y Krajcik (2012) para educación primaria. Se adoptó una escala de 1 a 7 , agrupando el puntaje en 5 niveles, a saber: 
- Sin respuesta: No se produce ninguna explicación; se asigna 0 puntos a la organización discursiva.

- Nivel A: En la afirmación, solo se menciona el proceso y/o los participantes sin agregar nueva información más allá del fenómeno científico presente en la pregunta científica. Las producciones calificadas con un puntaje de 1 o 2 son asignadas a este nivel (v.g. "Se junta", "El antebrazo y el brazo" y "El brazo se mueve", "La materia cambia de estado sólido a líquido").

- Nivel B: La explicación contiene una idea que podría responder a la pregunta de la tarea, pero no se incluyen evidencias. Las producciones calificadas con un puntaje de 3 o 4 son asignadas a este nivel (v.g. "Se juntan con el codo", "el brazo y el antebrazo se juntan con el codo"; "De estado sólido a líquido cambia porque el sólido se calienta”).

- Nivel C: En esta explicación se propone una idea en la afirmación y se introduce una evidencia. Las producciones calificadas con un puntaje de 5 o 6 son asignadas a este nivel (v.g. "Con los huesos y los ligamentos y los tendones. Como el modelo que hicimos y vimos", "Se juntan con los huesos y los ligamentos y los tendones. Como el modelo que hicimos y vimos").

- Nivel D: El estudiante cumple con las demandas epistémicas que supone la producción de una explicación científica, la cual presenta la totalidad de las fases discursivas. Las producciones calificadas con un puntaje de 7 son asignadas a este nivel porque se identifica una afirmación que contiene el proceso científico y la idea propuesta y, además, se incorpora una evidencia (e.g. "El brazo y el antebrazo se juntan mediante el codo porque lo une un hueso con otro hueso si no tuviéramos el codo no juntaríamos el brazo con el antebrazo como lo vimos en el modelo del brazo y el antebrazo en donde se juntan con los conectores que tiene el hueso", "Una materia cambia de estado sólido a líquido a causa del calor absorbido por el cuerpo a menor temperatura. Basado en el experimento cuando el objeto a menor temperatura está cerca del hielo absorbe el calor del objeto y se derrite").

\subsection{Recursos léxico-gramaticales asociados a la escritura}

Se calculó la frecuencia de aparición de diversos recursos asociados a la escritura (Beers \& Nagy, 2009; McNamara et al., 2010; Uccelli et al., 2013; Salas et al., 2016) a través de CLAN (Computerized Language Analysis) (MacWhinney, 2000). Se calcularon algunas de las mediciones frecuentemente utilizadas en estudios de escritura.

(1) Longitud (total de palabras): se refiere al total de palabras producidas en un texto. Para determinar la productividad, se contabilizó la cantidad de palabras de cada explicación. Los resultados de esta dimensión se reportan como la media del total de palabras por explicación científica. 
(2) Longitud (total de cláusulas): las explicaciones se segmentaron en cláusulas (Aravena et al., 2016; Meneses et al., 2018). En el presente estudio, hemos adoptado la definición operativa de Berman y Slobin, (1994), entendiendo a estas como unidades predicativas que expresa una situación única, ya sea esta una actividad, un evento o un estado (Berman \& Slobin, 1994). Adicionalmente, se realizó un proceso de doble segmentación en cláusulas del $20 \%$ de las explicaciones. Luego, se calculó el índice Kappa de Cohen, obteniendo un resultado de 0,88 , lo cual indica la confiabilidad de la segmentación. Los resultados reportados corresponden a la media de cláusulas por explicación científica.

(3) Diversidad léxica: esta dimensión corresponde a la cantidad de palabras léxicas de un texto (Gómez, Sotomayor, Bedwell, Domínguez \& Jéldrez, 2016). La diversidad léxica de las producciones fue medida dividiendo el total de palabras distintas (types) por el total de sus apariciones en el texto (tokens). Esta proporción fue controlada de acuerdo a la longitud de cada texto mediante la fórmula CTTR (corrected type/token ratio) (Malvern, Richard, Chipere \& Durán 2004; Gómez et al., 2016; Meneses et al., 2018). Para esta investigación no se utilizó VocD, ya que esta medición requiere de un mínimo de 50 palabras distintas por texto para ser calculada.

(4) Complejidad sintáctica: esta dimensión fue medida en número de palabras por cláusula (Beers \& Nagy, 2009).

\subsection{Recursos léxico-gramaticales del lenguaje académico}

Para responder a las preguntas de la presente investigación se identificaron los siguientes recursos propios del lenguaje académico y estos se reportan como proporción de cada recurso en el total de palabras.

(1) Vocabulario académico: corresponde al conjunto de palabras usadas más frecuentemente a lo largo de distintas disciplinas. Puesto que se las emplea para definir y explicar conceptos disciplinares, su significado es más específico y abstracto que aquellas del lenguaje cotidiano. Son de uso frecuente en contextos de educación formal (Crosson, Matsumura, Correnti \& Arlotta-Guerrero, 2012; Olinghouse \& Wilson, 2013). Palabras como 'sistema' y 'objeto' son ejemplos ilustrativos de este grupo.

(2) Vocabulario científico: corresponden a aquellas palabras específicas de una determinada área de la ciencia (Fang, 2004; Ávalos et al., 2017). En el caso de aquellas palabras con acepciones que podían originar lecturas ambiguas, cada una se revisó en su contexto de aparición y se desambiguó. Se consideraron como vocabulario científico términos como 'ligamento', 'músculo', 'energía'.

(3) Nominalizaciones: corresponde a un proceso de derivación morfológica de sustantivos a partir de verbos o adjetivos, que permite sistematizar y sintetizar 
información (Fang, 2004; Schleppegrell, 2004). Palabras como 'calentamiento', 'cambio', 'movimiento' son ejemplos ilustrativos de este grupo.

(4) Nexos causales: en sentido amplio, corresponden a piezas gramaticales que expresan relaciones de causalidad entre sintagmas, cláusulas y oraciones (Fang, 2004; Seah 2015; Ávalos et al., 2017; Meneses et al., 2018). Ejemplos de nexos causales son 'porque', 'debido a', 'ya que', 'gracias a', entre otros.

Para determinar la presencia de los recursos anteriormente descritos, se crearon listados de palabras y posteriormente fueron codificadas en las categorías mencionadas. Antes de analizar la frecuencia de estos recursos en el corpus, la codificación fue revisada por tres expertas.

\subsection{Plan de análisis}

Para responder la primera pregunta de esta investigación, se calcularon los estadísticos descriptivos de la organización discursiva y los distintos recursos léxicogramaticales de las explicaciones producidas por los estudiantes de la muestra. Para realizar las comparaciones entre dos grupos de muestras independientes, se aplicaron pruebas ' $\mathrm{t}$ ' (para sexo y tema), mientras que para comparar grupos de tres muestras independientes (para GSE del establecimiento) se aplicó el procedimiento estadístico ANOVA. Ambos recursos estadísticos tienen la finalidad de identificar las diferencias significativas entre grupos. Además, se aplicó la prueba post-hoc de Bonferroni para identificar, específicamente, los grupos que presentan diferencias significativas. Por último para responder a la segunda pregunta de investigación, se realizó un análisis de correlación múltiple con el fin de establecer las relaciones entre los distintos recursos discursivos y lingüísticos.

\section{Resultados}

La Tabla 1 presenta los resultados obtenidos de los análisis descriptivos (media y desviación estándar) para la organización discursiva, los recursos léxico-gramaticales asociados a la escritura y los específicos de lenguaje académico. 
Tabla 1. Estadísticos descriptivos para la organización discursiva, los recursos léxicogramaticales asociados a la escritura y los específicos de lenguaje académico en explicaciones científicas producidas por estudiantes chilenos de $4^{\circ}$ básico.

\begin{tabular}{|l|c|c|c|c|c|c|c|c|c|c|c|c|c|}
\hline & \multicolumn{4}{|c|}{ GSE Bajo } & \multicolumn{4}{c|}{ GSE Medio } & \multicolumn{5}{c|}{ GSE Alto } \\
\hline & Cambio & Movimiento & Cambio & \multicolumn{2}{|c|}{ Movimiento } & \multicolumn{2}{c|}{ Cambio } & \multicolumn{2}{c|}{ Movimiento } \\
\hline & $\mathbf{M}=\mathbf{5 1}$ & $\mathbf{D E}$ & $\mathbf{M}=\mathbf{5 6}$ & $\mathbf{D E}$ & $\mathbf{M}$ & $\mathbf{D E}$ & $\mathbf{M}$ & $\mathbf{D E}$ & $\mathbf{M}$ & $\mathbf{D E}$ & $\mathbf{M}$ & $\mathbf{D E}$ \\
\hline & 3,57 & 0,88 & 3,66 & 0,75 & 3,12 & 0,32 & 3,20 & 0,40 & 3,46 & 0,83 & 3,31 & 0,51 \\
\hline $\begin{array}{l}\text { Organización } \\
\text { discursiva }\end{array}$ & 13,9 & 8,02 & 9,23 & 4,29 & 7,83 & 4,65 & 6,84 & 4,6 & 11,12 & 7,02 & 11,0 & 7,46 \\
\hline $\begin{array}{l}\text { Longitud } \\
\text { (palabras) }\end{array}$ & 2,57 & 1,50 & 2,02 & 1,07 & 1,70 & 0,93 & 1,44 & 0,81 & 2,23 & 1,34 & 2,27 & 1,47 \\
\hline $\begin{array}{l}\text { Longitud } \\
\text { (cláusulas) }\end{array}$ & 6,25 & 1,51 & 5,40 & 1,17 & 5,03 & 1,38 & 4,50 & 1,42 & 5,66 & 1,74 & 5,45 & 1,75 \\
\hline $\begin{array}{l}\text { Diversidad } \\
\text { léxica }\end{array}$ & 5,68 & 2,08 & 5,01 & 2,25 & 4,57 & 1,47 & 4,77 & 2,23 & 4,97 & 2,01 & 4,96 & 2,17 \\
\hline $\begin{array}{l}\text { Complejidad } \\
\text { sintáctica }\end{array}$ & 0,08 & 0,06 & 0,14 & 0,11 & 0,09 & 0,09 & 0,05 & 0,08 & 0,12 & 0,09 & 0,10 & 0,09 \\
\hline $\begin{array}{l}\text { Vocabulario } \\
\text { académico \% }\end{array}$ & 0,16 & 0,11 & 0,17 & 0,16 & 0,15 & 0,13 & 0,36 & 0,16 & 0,15 & 0,16 & 0,24 & 0,15 \\
\hline $\begin{array}{l}\text { Vocabulario } \\
\text { científico \% }\end{array}$ & 0,02 & 0,04 & 0,00 & 0,02 & 0,01 & 0,04 & 0,01 & 0,04 & 0,05 & 0,15 & 0,02 & 0,04 \\
\hline $\begin{array}{l}\text { Nominalización } \\
\text { \% }\end{array}$ & 0,00 & 0,01 & 0,01 & 0,04 & 0,02 & 0,06 & 0,00 & 0,01 & 0,00 & 0,01 & 0,00 & 0,01 \\
\hline Nexo causal \%
\end{tabular}

En la Tabla 1, se presentan los resultados del análisis de la organización discursiva para las explicaciones científicas. Los puntajes oscilan entre 3,12 y 3,66; por lo tanto, se sitúan en el nivel B. Esto quiere decir que en las explicaciones se identifica una idea para contestar a la pregunta científica, pero no incluyen evidencias que la sustenten. En la Tabla 2 se presentan ejemplos prototípicos de explicaciones producidas por estudiantes pertenecientes a distintos grupos socioeconómicos.

Tabla 2. Ejemplos de explicaciones científicas de los tres grupos socioeconómicos (afirmación).

\begin{tabular}{|c|c|c|c|}
\hline GSE & \multicolumn{3}{|c|}{ Ejemplo } \\
\hline \multirow{2}{*}{$\begin{array}{l}\text { GSE } \\
\text { bajo }\end{array}$} & [El brazo] [se mueve] & $\begin{array}{r}\text { [por la articulación, si } \\
\text { pod }\end{array}$ & $\begin{array}{l}\text { emos articulaciones no nos } \\
\text { mover.] }\end{array}$ \\
\hline & (fenómeno científico) & \multicolumn{2}{|c|}{ (idea propuesta) } \\
\hline \multirow{2}{*}{$\begin{array}{l}\text { GSE } \\
\text { medio }\end{array}$} & Lo que permite que & [el brazo] [se mueva] & $\begin{array}{c}\text { [son los nervios, los huesos, y } \\
\text { músculos.] }\end{array}$ \\
\hline & & (fenómeno científico) & (idea propuesta) \\
\hline \multirow{2}{*}{$\begin{array}{c}\text { GSE } \\
\text { alto }\end{array}$} & Lo que permite que & [el brazo] [se mueva] & $\begin{array}{c}\text { [son las articulaciones y los } \\
\text { huesos.] }\end{array}$ \\
\hline & & (fenómeno científico) & (idea propuesta) \\
\hline
\end{tabular}

En la Tabla 2 se puede observar que las explicaciones científicas están formadas por la fase correspondiente a la afirmación; por lo tanto, se menciona el fenómeno científico y se propone una idea para responder a la pregunta científica. Por lo tanto, al producir una explicación científica, los estudiantes de esta muestra, que no fueron 
expuestos a una enseñanza explícita de la explicación científica, construyen afirmaciones para contestar a la pregunta científica propuesta; sin embargo, no sustentan esas ideas en datos obtenidos a través de la experimentación o la construcción de modelos.

Con respecto a los recursos léxico-gramaticales asociados a la escritura, se puede observar que según la 'longitud-palabras', las producciones son breves con un promedio de 10 palabras con una fluctuación entre 7 y 14 palabras aproximadamente. Si se considera la 'longitud-cláusulas', se observa que en promedio se identifican 2 cláusulas en las explicaciones producidas; esto quiere decir que se pueden reconocer dos unidades con predicado unificado, lo que puede relacionarse con el tipo de pregunta científica presentada.

En cuanto a la diversidad léxica, en términos generales, los estudiantes de la muestra usan en promedio 5,3 palabras distintas por explicación, lo que da cuenta de un repertorio léxico acotado pero diverso si contamos que la extensión promedio de las explicaciones es de 10 palabras. Respecto a la complejidad sintáctica, en la muestra se observa un promedio de 5 palabras por cláusula; de este modo se observa que si bien hay en promedio dos cláusulas por explicación, estas son breves y no extienden las ideas para dar cuenta del mecanismo que produce el proceso científico por el cual se pregunta. El siguiente ejemplo, de dos cláusulas y un total de seis palabras, ilustra las medias de los recursos mencionados:

\section{[Cuando se derrite] [ocurre la fusión] Cláusula $1 \quad$ Cláusula 2}

En cuanto a los recursos de lenguaje académico, se observa que en promedio un $10 \%$ de las palabras de las explicaciones corresponde a vocabulario académico mientras que el $21 \%$ corresponde a vocabulario científico. Por lo tanto, si bien las producciones son breves un porcentaje relevante de palabras corresponden al registro académico. Con respecto a las nominalizaciones y los nexos causales, se identificaron en promedio un $2 \%$ y un $1 \%$ respectivamente.

\subsection{Diferencias según sexo de los estudiantes}

Para comparar los resultados obtenidos según sexo, se realizó una prueba de muestras independientes, cuyos resultados mostraron que si bien no se encontraron diferencias significativas para la organización discursiva, sí se detectaron diferencias significativas en tres recursos asociados a la escritura: longitud-palabras, diversidad léxica y longitud-cláusulas. La longitud-palabras de las explicaciones científicas producidas por hombres y mujeres presentó diferencias significativas $(\mathrm{t}(324)=-2,573$, $p<, 05)$. En los hombres, la media fue de 9,08 palabras por explicación, mientras que las mujeres obtuvieron una media de 10,94 palabras por explicación. También se 
constataron diferencias significativas en diversidad léxica, puesto que las mujeres mostraron utilizar una variedad de palabras levemente mayor en comparación con los hombres; sin embargo, dicha diferencia es significativa $(\mathrm{t}(324)=-2,255, p<, 05)$. En el caso de los hombres, la media fue de 5,19 palabras distintas por producción, mientras que en el de las mujeres, 5,59. Finalmente, se constataron diferencias significativas en la longitud-cláusulas utilizadas $(\mathrm{t}(324)=-2.612, p<, 05)$, pues la media de los hombres fue de 1,86, mientras que la de las mujeres 2,23. De estos resultados se desprende que si bien las diferencias son pequeñas entre hombres y mujeres, estas son significativas, lo que muestra que las mujeres producen explicaciones un poco más extensas y con una diferencia mínima de palabras diversas. No obstante, no se encontraron diferencias significativas en el uso de los recursos del lenguaje académico que son relevantes para la producción de explicaciones científicas, es decir, vocabulario académico, vocabulario científico, nominalizaciones y nexo causal.

\subsection{Diferencias según grupo socioeconómico}

Respecto a los resultados obtenidos de acuerdo a la variable socioeconómica, se encontraron diferencias significativas entre grupos. El análisis ANOVA identificó que dentro del nivel B alcanzado por todas las explicaciones producidas por los estudiantes, los niños y niñas de los colegios con GSE bajo y alto producen afirmaciones un poco más completas que los de GSE medio debido a que tienden a nombrar el fenómeno científico completo $(F(2,323)=14,254, p<, 05)$.

Un patrón similar se observa en la longitud-palabras y longitud-cláusulas en la prueba ANOVA que determinó diferencias significativas en el número total de palabras de los estudiantes de GSE bajo y alto en comparación con el de GSE medio $(F(2,323)=14,645, p<, 05)$ y en el número total de cláusulas $(F(2,323)=12.163, p<$ ,05). Esto quiere decir que si bien todas las producciones son breves, aquellas de los estudiantes del GSE medio tienden a ser menos extensas en comparación con las de los estudiantes de los otros dos colegios.

En cuanto a la diversidad léxica, los resultados indican que existen diferencias significativas entre el GSE medio con los GSE bajo y alto $(F(2,323)=13,874, p<$ ,05). Esto quiere decir que los estudiantes del colegio de GSE medio producen explicaciones científicas con un menor número de palabras distintas en comparación a las producidas por los estudiantes de los otros dos establecimientos. No se encontraron diferencias significativas en la complejidad sintáctica entre grupos.

Con respecto a los recursos léxico-gramaticales propios del lenguaje académico, se obtuvieron resultados similares para el vocabulario académico. La prueba ANOVA mostró que en las producciones de los estudiantes de GSE bajo y alto hay una proporción significativamente más alta de palabras académicas que en las producidas por los estudiantes de GSE medio $(F(2,323)=7,813, p<, 005)$. En cambio, se identificaron diferencias significativas en vocabulario científico en las producciones de 
estudiantes de GSE medio, es decir, se identificó una mayor proporción de palabras científicas en comparación con las producidas por estudiantes de los otros dos establecimientos $(F(2,323)=7.355, p<, 005)$. En cuanto a las nominalizaciones, si bien tienen una baja aparición en las explicaciones producidas, los estudiantes del GSE alto las utilizan significativamente más que los estudiantes de los otros establecimientos $(F(2,323)=4,81, p<, 005)$. Por último, no se encontraron diferencias en el uso de nexos causales entre grupos.

\subsection{Diferencias según tema}

Los resultados obtenidos a partir de la prueba de muestras independientes para la comparación de grupos por temas de las explicaciones indican que no existen diferencias significativas en el caso de la organización discursiva. En los recursos asociados a la escritura, se encontraron diferencias significativas en la longitudpalabras y la diversidad léxica. Los resultados muestran que las explicaciones sobre el tema 'cambio de estado de la materia' poseen una media significativamente más alta en el caso de la longitud-palabras $(\mathrm{t}(324)=2,51, p<0,13)$ con una media de 10,95 palabras, mientras que el tema 'movimiento del brazo' tiene una media promedio de 9,02 palabras por explicación. En el caso de la diversidad léxica, se observan diferencias a favor del tema 'cambio de estado de la materia' ( $\mathrm{t}(324)=2,89, p<0,004)$; la media de este tema es de 5,65 palabras léxicas distintas por explicación, mientras que en el tema 'movimiento del brazo' la media es de 5,12. No se encontraron diferencias significativas en la longitud-cláusulas ni en la complejidad sintáctica.

En cuanto a los recursos léxicogramaticales académicos, se halló una diferencia significativa en el uso del vocabulario científico por tema $(\mathrm{t}(324)=-5,85, p<0,000)$, pues la media de este recurso en el tema 'cambio de estado de la materia' corresponde al $15 \%$ de las palabras propias de las explicaciones científicas, mientras que en el caso del tema 'movimiento del brazo' la media es de un $26 \%$ de las palabras producidas para esta explicación. No se encontraron diferencias significativas en vocabulario académico, nominalizaciones ni nexo causal. En la Tabla 3, se puede observar explicaciones que ilustran las diferencias entre temas.

Tabla 3. Ejemplos de explicaciones y uso de vocabulario científico de acuerdo al tema producidas por un mismo estudiante.

\begin{tabular}{|l|l|}
\hline \multicolumn{1}{|c|}{ Tema 'cambio de estado de la materia' } & \multicolumn{1}{c|}{ Tema 'movimiento del brazo' } \\
\hline $\begin{array}{l}\text { Cuando un cuerpo sólido se derrite y queda líquido } \\
\text { como cuando un helado se derrite. }\end{array}$ & $\begin{array}{l}\text { El sistema locomotor que está compuesto por } \\
\text { huesos que son estructuras rígidas, articulaciones } \\
\text { zona de unión entre huesos que permite el } \\
\text { movimiento de músculos órganos internos que } \\
\text { permite el movimiento y tendones une a los huesos } \\
\text { y músculos. }\end{array}$ \\
\hline
\end{tabular}




\subsection{Correlaciones entre organización discursiva, recursos léxico-gramaticales asociados a la escritura y recursos de lenguaje académico}

Para responder la segunda pregunta de investigación sobre las relaciones entre la organización discursiva, los recursos léxico-gramaticales asociados a la escritura y aquellos propios del lenguaje académico se aplicó un método estadístico de correlaciones.

Tabla 4. Correlaciones entre la organización discursiva, recursos léxicogramaticales asociados a la escritura y específicos del lenguaje académico en explicaciones científicas.

\begin{tabular}{|c|c|c|c|c|c|c|c|c|c|}
\hline & 1 & 2 & 3 & 4 & 5 & 6 & 7 & 8 & 9 \\
\hline $\begin{array}{l}\text { 1. Organización } \\
\text { discursiva }\end{array}$ & 1 & & & & & & & & \\
\hline 2. Longitud (palabras) &, $501^{* *}$ & 1 & & & & & & & \\
\hline 3. Longitud (cláusulas) &, $495^{* *}$ &, $884^{* *}$ & 1 & & & & & & \\
\hline 4. Diversidad léxica & $486^{* *}$ &, $900^{* *}$ &, $779^{* *}$ & 1 & & & & & \\
\hline $\begin{array}{l}\text { 5. Complejidad } \\
\text { sintáctica }\end{array}$ & ,098 &, $339^{* *}$ &,- 071 &, $424^{* *}$ & 1 & & & & \\
\hline $\begin{array}{l}\text { 6. Vocabulario } \\
\text { académico }\end{array}$ &, $225^{* *}$ &, $253^{* *}$ &, $301^{* *}$ &, $342^{* *}$ & ,043 & 1 & & & \\
\hline $\begin{array}{l}\text { 7. Vocabulario } \\
\text { científico }\end{array}$ &,$- 253^{* *}$ &,$- 392^{* *}$ &,$- 386^{* *}$ &,$- 492^{* *}$ &,$- 156^{* *}$ &,$- 488^{* *}$ & 1 & & \\
\hline 8. Nominalización &,- 067 &,- 07 &,- 089 &,- 081 &,- 013 &,- 069 &,$- 111^{*}$ & 1 & \\
\hline 9. Nexo Causal &,- 04 &,- 015 &,- 044 &,- 012 &, 058 & $146^{* *}$ &,$- 139^{*}$ & ,019 & 1 \\
\hline
\end{tabular}

En la Tabla 4 se muestran las correlaciones entre las distintas mediciones. De acuerdo a los resultados, se observa que la organización discursiva correlaciona significativamente de manera positiva y moderada con la longitud-palabras, la longitud-cláusulas y la diversidad léxica y de manera baja con el vocabulario académico; en cambio, correlaciona significativamente de manera baja y negativa con el vocabulario científico. Además, la longitud-palabras correlaciona significativamente de manera positiva y fuerte con la longitud-cláusulas y con la diversidad léxica, mientras que dicha longitud con la complejidad sintáctica y con el vocabulario académico se correlaciona positivamente pero de manera moderada y baja, respectivamente. Existe también una correlación positiva, significativa y moderada entre la longitud-cláusulas y el vocabulario académico y entre la diversidad léxica y la complejidad sintáctica, así como con vocabulario académico. Por lo tanto, en la medida que la longitud de la explicación aumenta, también aumenta la presencia de los recursos léxico-gramaticales asociados a la escritura, la presencia de palabras propias del vocabulario académico, así como también se constata un mayor dominio de la organización discursiva.

Por otra parte, los resultados indican que existen correlaciones significativamente negativas entre el vocabulario científico y los otros recursos analizados e incluso con la 
organización discursiva. Estas correlaciones negativas son moderadas y bajas e indican que en la medida que los otros recursos aumentan, disminuye la presencia del vocabulario científico. Esto podría indicar que el vocabulario científico opera en un dominio diferente al lingüístico y estar más bien relacionado con el dominio conceptual para la construcción de explicación científica. Asimismo, la presencia de mayor vocabulario científico densifica la producción realizada haciendo que la explicación sea más compacta.

\section{DISCUSIÓN Y CONCLUSIONES}

Este artículo ha tenido por objetivo describir, comparar y relacionar la organización discursiva, los recursos léxico-gramaticales asociados a la escritura y aquellos propios del lenguaje académico en explicaciones científicas producidas por estudiantes de cuarto básico según sexo, grupo socioeconómico y tema científico. Los análisis lingüísticos de los datos y su procesamiento estadístico por medio de distintas pruebas ha mostrado que los estudiantes producen explicaciones científicas en las que desarrollan una afirmación con ideas para responder a la pregunta científica; sin embargo, no introducen evidencias como respaldo, fase fundamental de este género disciplinar. Estos resultados exploratorios muestran que los estudiantes de $4^{\circ}$ básico que participaron en el estudio más bien siguen un patrón-pregunta respuesta, vale decir, dan una respuesta más o menos completa a la pregunta formulada; por lo tanto, requieren de una enseñanza explícita de este género disciplinar para incorporar evidencias que sustenten las ideas que proponen.

La investigación de McNeill et al. (2006) con estudiantes de $7^{\circ}$ grado muestra que los estudiantes antes de una intervención producen explicaciones científicas en las que no introducen la evidencia; en cambio, una vez realizada la intervención que ofrece distintos andamiajes, los estudiantes logran construir explicaciones científicas incorporando datos científicos que sustenten sus ideas. Un resultado similar se encuentra con estudiantes más pequeños en McNeill y Krajcik (2012). Por lo tanto, los resultados que se reportan en este estudio muestran que estudiantes que no han recibido una enseñanza explícita sobre la construcción de explicaciones científicas tenderán más bien a contestar la pregunta científica sin buscar evidencias que sustenten sus ideas. La descripción realizada permite comprender cómo estudiantes chilenos $4^{\circ}$ básico responden a una tarea de ciencias y cómo se representan una explicación científica a partir del análisis de la organización discursiva. Como plantea el National Research Council (2012), las explicaciones científicas sustentadas en evidencia son escasamente enseñadas en la clase de ciencias; sin embargo, juegan un papel fundamental en la construcción del conocimiento científico. Desde esta perspectiva, los escolares requieren, entonces, de la enseñanza explícita sobre cómo producir una explicación científica para aprender no solo a acceder a los conocimientos propios de las disciplinas científicas sino también a los modos en que 
estos se construyen (McNeill et al., 2006; Krajcik \& Sutherland, 2010; McNeill \& Krajcik, 2012; Zembal-Saul et al., 2013).

En cuanto a los recursos léxicogramaticales asociados a la escritura, se puede destacar que las explicaciones producidas por los estudiantes de la muestra son breves, con una variedad de palabras importante al considerar su extensión y con una complejidad sintáctica que da cuenta de que las ideas que se empaquetan no son desarrolladas. Salas et al. (2016), al analizar narraciones producidas por estudiantes de primaria, encontraron que los textos son más extensos en comparación a los analizados en este estudio con un promedio de 75 palabras, con un número promedio de 14,6 cláusulas. En el estudio de Gómez et al. (2016), encontraron que los textos producidos por estudiantes de $4^{\circ}$ básico eran más extensos en todos los géneros analizados (narrativos 99,9 palabras, persuasivos, 49,5, informativos 46,7). En cambio, en el estudio de Meneses et al. (2018) se obtienen resultados similares a los obtenidos en este estudio al comparar los resultados en explicaciones producidas por estudiantes de $4^{\circ}$ básico con intervención al inicio del año escolar. Es decir, en dicha investigación, los estudiantes antes de recibir la enseñanza explícita producían en promedio explicaciones de 16 palabras, un poco más extensas que lo encontrado en estudio, con un número de cláusulas de 2,9. Con respecto a los recursos específicos del lenguaje académico, en el estudio de Meneses et al. (2018) se encontró una proporción similar de vocabulario académico (7\%), vocabulario científico $(17 \%)$, nominalización (1\%) y nexo causal (1\%). Por lo tanto, a partir de la revisión de la literatura y los resultados de esta investigación se observa que las explicaciones científicas producidas por los estudiantes son más breves en comparación con la producción de narraciones.

Respecto a las diferencias por grupo, se encontró que si bien las diferencias son pequeñas, las explicaciones producidas por mujeres son significativamente más extensas (longitud-palabras y longitud-cláusulas) y con una mayor diversidad léxica que las producidas por los hombres; sin embargo, no se encontraron diferencias en el uso de recursos de lenguaje académico ni en la organización discursiva. En relación con los resultados a partir del GSE, estos dan cuenta de que existen diferencias entre grupos, principalmente entre el GSE medio y los GSE bajo y alto. Las explicaciones producidas por los estudiantes de colegio de GSE medio son más breves, con una menor diversidad léxica y con una menor presencia de vocabulario académico; sin embargo, con una presencia significativamente mayor de vocabulario científico. En cuanto a las producciones de estudiantes de GSE alto, se diferencian significativamente por un mayor uso de las nominalizaciones, recurso de lenguaje académico que permite crear conceptos técnicos, establecer relaciones de causa y efecto, sintetizar información y nombrar objetos de la realidad (Ávalos et al., 2017; Schleppegrell, 2004). En el caso de los procesos científicos explicados, se observan diferencias significativas entre algunos recursos a favor del tema 'cambio de estado de 
la materia' (longitud-palabras y diversidad léxica). Estas podrían deberse a un mayor dominio conceptual por parte de los estudiantes sobre este contenido al momento de aplicación de las tareas analizadas (fin del año escolar).

Por último, se constató que los recursos tales como la longitud de palabras, la longitud de cláusulas, la complejidad sintáctica, el vocabulario académico y la organización discursiva están estrechamente vinculados. En cambio, el vocabulario científico correlaciona negativamente con todos los otros recursos analizados, lo que podría interpretarse como una medición más relacionada con el dominio conceptual que con el lingüístico. Por lo tanto, se requieren estudios posteriores para explorar a través de análisis factoriales o de componentes principales las relaciones entre los distintos recursos analizados.

Si bien la presente investigación ha aportado con evidencia sobre la escritura disciplinar de estudiantes chilenos de $4^{\circ}$ básico, investigaciones con una muestra mayor y que abarquen distintos niveles escolares permitirían una comprensión más cabal de las demandas de la literacidad disciplinar (Shanahan \& Shanahan, 2008). Asimismo, otra limitación de este estudio dice relación con la no inclusión de criterios para determinar la calidad de la explicación científica. Las futuras investigaciones sobre escritura disciplinar que incluyan mediciones de calidad y la contribución específica de variables de dominio conceptual y lingüístico son necesarias en español para construir interpretaciones que vayan más allá del capital cultural y muestren cómo la escuela aproxima a los estudiantes a un registro y a géneros específicos a través de los cuales se construye el conocimiento.

Esta investigación aporta evidencia sobre qué recursos discursivos y lingüísticos usan los estudiantes chilenos de $4^{\circ}$ básico en una tarea, cuyo propósito es la explicación de un proceso científico sin ofrecer andamiajes ni enseñanza explícita. La escritura de explicaciones, entonces, no es solo una herramienta de aprendizaje y evaluación, sino también una práctica científica que requiere ser enseñada desde una perspectiva lingüística y disciplinar (McNeill et al., 2006; Seah et al., 2011; Brisk, 2015; Seah, 2015). El compromiso por el desarrollo del lenguaje académico y de las habilidades de escritura incumbe no solo a los docentes del área de lenguaje sino a las demás especialidades: cada disciplina construye sus géneros como formas particulares de razonamiento a través de recursos lingüísticos específicos (Seah et al., 2011; Brisk, 2015; Seah, 2015;). La tarea de enseñar a escribir a través de las disciplinas es una tarea de los profesores de las distintas áreas puesto que la construcción de conocimiento está mediada por el dominio de un lenguaje y de unos géneros discursivos específicos en los que se despliegan los modos de razonamiento disciplinar (Bazerman et al., 2016; Grøver et al., 2019; Uccelli, 2019). Como plantea Bazerman et al. (2016), una parte fundamental del proceso de enculturación es especificar las expectativas que configuran los distintos géneros discursivos. Este estudio contribuye realizando una 
descripción lingüística del género explicación para orientar pedagógicamente intervenciones en el área de ciencias y para comprender algunos de los recursos discursivos y lingüísticos que los estudiantes usan al construir una explicación científica.

\section{REFERENCIAS BIBLIOGRÁFICAS}

Agencia de Calidad de la Educación. (2013). Metodología de construcción de grupos socioeconómicos: Pruebas SIMCE 2012 [en línea]. Disponible en: http://archivos.agenciaeducacion.cl/Metodologia-de-Construccion-deGrupos-Socioeconomicos-SIMCE-2012.pdf [Consulta: 16 de enero de 2019].

Agencia de Calidad de la Educación (2017). Informe de resultados de aprendizaje escritura 2016. Sexto Básico. Santiago, Chile: Ministerio de Educación [en línea]. Disponible en:

http://archivos.agenciaeducacion.cl/resultados nacionales escritura 2016.pd f.

Andrade, V., Freire, S. \& Baptista, M. (2017). Constructing scientific explanations: A system of analysis for students' explanations. Research in Science Education, 49(3), 787-807. DOI:10.1007/s11165-017-9648-9

Aravena, S. \& Hugo, E. (2016). Desarrollo de la complejidad sintáctica en textos narrativos y explicativos escritos por estudiantes secundarios. Lenguas Modernas, 47, 9-40.

Aravena, S., Figueroa, J., Quiroga, R. \& Hugo, E. (2016). Organización discursiva de dos géneros en estudiantes de tres niveles de escolaridad y diferentes grupos sociales. Revista Signos. Estudios de Lingüistica, 49(91), 168-191. DOI: 10.4067/S0718-09342016000200002

Ávalos, M., Gómez, M., Gort, M. \& Secada, W. (2017). Hey! today i will tell you about the water cycle!: Variations of language and organizational features in thirdgrade science explanation writing. The Elementary School Journal, 118(1), 149176. DOI: $10.1086 / 692987$

Bañales, G., Ahumada, S., Martínez, R., Martínez, M. \& Messina, P. (2018). Investigaciones de la escritura en la educación básica en Chile: Revisión de una década (2007-2016). RLA, 56(1), 59-84.

Bazerman, Ch., Little, J., Bethel, L., Chavkin, T., Fouquette, D. \& Garufis, J. (2016). Escribir a través del curriculum: Una guía de referencia (Trad. V. de F. Navarro). Córdoba: Universidad Nacional de Córdoba. 
Beers, S. F. \& Nagy, W. E. (2009). Syntactic complexity as a predictor of adolescent writing quality: Which measures? Which genre? Reading and Writing, 22(2), 185200. DOI: $10.1007 / \mathrm{s} 11145-007-9107-5$

Berman, R. \& Ravid, D. (2009). Becoming a literate language user: Oral and written text construction across adolescence. En D. Olson \& N. Torrance (Eds.), The Cambridge handbook of literacy (pp. 92-111). Nueva York: Cambridge University Press.

Berman, B. \& Slobin, D. (1994). Relating events in narrative: A crosslinguistic developmental study. Mahwah: Lawrence Erlbaum.

Brisk, M. E. (2015). Engaging students in academic literacies. Genre-based pedagogy for K-5 classroom. Nueva York: Routledge.

Bronckart, J. P. (2004). Secuencias y otras formas de planificación. En J. P. Bronckart (Ed.), Actividad verbal, textos y discursos. Por un interaccionismo sociodiscursivo (pp. 135-153). Madrid: Fundación infancia y Aprendizaje.

Crosson, A., Matsumura, L., Correnti, R. \& Arlotta-Guerrero, A. (2012). The quality of writing tasks and students' use of academic language in Spanish. The Elementary School Journal, 112(3), 469-496. DOI: $\underline{10.1086 / 663298}$

Fang, Z. (2004). Scientific literacy: A systemic functional linguistics perspective. Science Education, 89(2), 335-347. DOI: $10.1002 /$ sce.20050

Fang, Z. (2014). Writing a report: A study of preadolescents' use of informational language. Linguistics and the Human Sciences, 10(2), 103-131. DOI: 10.1558/lhs.v10.2.28556

Gómez, G., Sotomayor, C., Bedwell, P., Domínguez, A. M. \& Jéldrez, E. (2016). Analysis of lexical quality and its relation to writing quality for $4^{\text {th }}$ grade, primary school students in Chile. Reading and Writing, 29(7), 1317-1336. DOI $\underline{10.1007 / s 11145-016-9637-9}$

Graham, S., Gillespie, A. \& McKeown, D. (2013). Writing: Importance, development, and instruction. Reading and Writing, 26(1), 1-15. DOI: 10.1007/s11145-0129395-2

Grøver, V., Uccelli, P., Rowe, M. L. \& Lieven, E. (2019). Learning through language. En V. Grøver, P. Uccelli, M. L. Rowe \& E., Lieven (Eds.), Learning through language. Towards an educationally informed theory of language learning (pp. 1-16), Cambridge: Cambridge University Press. 
Ibáñez, R. (2010). El texto disciplinar en la transmisión del conocimiento especializado. Estudios Filológicos, 46, 59-80. DOI: 10.4067/S007117132010000200004

Krajcik, J. S. \& Sutherland, L. M. (2010). Supporting students in developing literacy in Science. Science, 328(5977), 456-459. DOI: $10.1126 /$ science.1182593

MacWhinney, B. (2000). The CHILDES Project: Tools for analyzing talk. Mahwah, NJ: Lawrence Erlbaum.

Malvern, D., Richards, B., Chipere, N. \& Durán, P. (2004). Lexical diversity and language development: Quantification and assessment. Hampshire: Palgrave Macmillan.

McNamara, D., Crossley, S. A. \& McCarthy, P. M. (2010). Linguistic features of writing quality. Written Communication, 27(1), 57-86. DOI: $\underline{10.1177 / 0741088309351547}$

McNeill, K. \& Krajcik, (2012). Supporting grade 5-8 students in constructing explanations in Science. The claim, evidence, and reasoning framework for talk and writing. Boston, MA: Pearson Education.

McNeill, K., Lizotte, J., Krajcik, J. \& Marx, R., (2006). Supporting students' construction of scientific explanations by fading scaffolds in instructional materials. The Journal of the Learning Sciences, 15(2), 153-191. DOI: $10.1207 / \mathrm{s} 15327809 j \mathrm{j} 15021$

Meneses, A., Hugo, E., Montenegro, M., Valenzuela, A. \& Ruiz, M. (2018). Explicaciones científicas: propuestas para la enseñanza del lenguaje académico. Boletín de Lingüística, XXX(49-50), 134-157.

Miller, B. \& McCardle, P. (2011). Reflections on the need for continued research on writing. Reading \& Writing, 24(2), 121-132. DOI: 10.1007/s11145-010-9267-6

Miras, M. (2000). La escritura reflexiva: Aprender a escribir y aprender acerca de lo que se escribe. Infancia y Aprendizaje, 23(89), 65-80. DOI: $\underline{10.1174 / 021037000760088099}$

National Research Council (2012). A framework for K-12 science education: Practices, crosscutting concepts, and core ideas. Committee on a conceptual framework for new K-12 science education standards. Board on Science Education, Division of Behavioral and Social Sciences and Education. Washington, DC: The National Academies Press.

Olinghouse, N. \& Wilson, J. (2013). The relationship between vocabulary and writing quality in three genres. Reading and Writing, 26(1), 45-65. DOI: $\underline{10.1007 / \mathrm{s} 11145-012-9392-5}$ 
Ravid, D. \& Tolchinsky, L. (2002). Developing linguistic literacy: A comprehensive model. Journal of Child Language, 29(2), 417-447. DOI: $\underline{10.1017 / \mathrm{S} 0305000902009169}$

Ruiz, M., Meneses, A., Hugo, E. \& Rubio, A. (2018). Manual de segmentación de explicaciones cientificas en fases discursivas. Manuscrito no publicado.

Salas, N., Llauradó, A., Castillo, C., Taulé, M. \& Martí, M. A. (2016). Linguistic correlates of text quality from childhood to adulthood. En J. Perera, M. Aparici, E. Rosado \& N. Salas (Eds.), Written and spoken language development across the lifespan. Essays in honour of Liliana Tolchinsky (pp. 307-326). Nueva York: Springer.

Schleppegrell, M. J. (2004). The language of schooling: A functional linguistic perspective. Londres: Lawrence Erlbaum.

Schleppegrell, M. J. (2012). Academic language in teaching and learning. Introduction to the Special Issue. The Elementary School Journal, 112(3), 409-418. DOI: $\underline{10.1086 / 663297}$

Seah, L. H. (2015). Understanding the conceptual and language challenges encountered by grade 4 students when writing scientific explanations. Research in Science Education. 46(3), 413-437. DOI:10.1007/s11165-015-9464-z

Seah, L. H., Clarke, D. \& Hart, C. (2011). Understanding students' language use about expansion through analyzing their lexicogrammatical resources. Science Education 95, 852-876. DOI: $10.1002 /$ sce.20448

Shanahan, T. \& Shanahan, C. (2008). Teaching disciplinary literacy to adolescents: Rethinking content area literacy. Harvard Educational Review, 78(1), 40-59. DOI: 10.17763/haer.78.1.v62444321p602101

Snow, C. \& Uccelli, P. (2009). The challenge of academic language. En D. R. Olson \& N. Torrance (Eds.), The Cambridge handbook of literacy (pp. 112-133). Nueva York: Cambridge University Press.

Sotomayor, C., Gómez, G., Jéldrez, E., Bedwell, P., Domínguez, A. M. \& Ávila, N. (2016). Evaluación analítica de la escritura de estudiantes de $4^{\circ}$ año básico en Chile. Onomázein, 34, 381-425. DOI: 10.7764/onomazein.34.21

Uccelli, P. (2019). Learning the language for school literacy. En V. Grøver, P. Uccelli, M. L. Rowe \& E. Lieven (Eds.), Learning through language. Towards an educationally informed theory of language learning, (pp. 95-109) Cambridge: Cambridge University Press. 
Uccelli, P. Dobbs, C. L. \& Scott, J. (2013). Mastering academic language: Organization and stance in the persuasive writing of high school students. Written Communication, 30(1), 36-62. DOI: 10.1177/0741088312469013

Uccelli, P., Phillips Galloway, E. P., Barr, C. D., Meneses, A. \& Dobbs, C. L. (2015). Beyond vocabulary: Exploring cross-disciplinary academic-language proficiency and its association with reading comprehension. Reading Research Quarterly, 50(3), 337-356. DOI: 10.1002/rrq.104

Zembal-Saul, C., McNeill, K. \& Hershberger, K. (2013). What's your evidence? Engaging K-5 students in constructing explanations in Science. Boston, MA: Pearson Education.

\section{* AGRADECIMENTO}

Las autoras desean expresar su agradecimiento a Marcela Ruiz, Evelyn Hugo, Andrea Valenzuela, Valentina Basso y Diego Torrealba por la colaboración que brindaron para desarrollo de esta investigación.

Este artículo contó con el apoyo de CONICYT, FONDECYT REGULAR 1150238 y FONDECYT REGULAR 1200882. 